\title{
Invisible neutrino decay in the light of NOvA and T2K data
}

\author{
Sandhya Choubey, ${ }^{a, b}$ Debajyoti Dutta $^{c}$ and Dipyaman Pramanik ${ }^{a}$ \\ ${ }^{a}$ Harish-Chandra Research Institute, HBNI, \\ Chhatnag Road, Jhunsi, Allahabad, 211019 India \\ ${ }^{b}$ Department of Physics, School of Engineering Sciences, KTH Royal Institute of Technology, \\ AlbaNova University Center, Stockholm, 10691 Sweden \\ ${ }^{c}$ Assam Don Bosco University, Tapesia Campus, \\ Sonapur, Assam, 782402 India \\ E-mail: sandhya@hri.res.in, debajyoti.dutta@dbuniversity.ac.in, \\ dipyamanpramanik@hri.res.in
}

ABSTRACT: We probe for evidence of invisible neutrino decay in the latest NOvA and T2K data. It is seen that both NOvA and T2K data sets are better fitted when one allows for invisible neutrino decay. We consider a scenario where only the third neutrino mass eigenstate $\nu_{3}$ is unstable and decays into invisible components. The best-fit value for the $\nu_{3}$ lifetime is obtained as $\tau_{3} / m_{3}=3.16 \times 10^{-12} \mathrm{~s} / \mathrm{eV}$ from the analysis of the NOvA neutrino data and $\tau_{3} / m_{3}=1.0 \times 10^{-11} \mathrm{~s} / \mathrm{eV}$ from the analysis of the T2K neutrino and anti-neutrino data. The combined analysis of NOvA and T2K gives $\tau_{3} / m_{3}=5.01 \times 10^{-12} \mathrm{~s} / \mathrm{eV}$ as the best-fit lifetime. However, the statistical significance for this preference is weak with the no-decay hypothesis still allowed at close to $1.5 \sigma$ C.L. from the combined data sets, while the two experiment individually are consistent with no-decay even at the $1 \sigma$ C.L. At $3 \sigma$ C.L., the NOvA and T2K data give a lower limit on the neutrino lifetime of $\tau_{3} / m_{3}$ is $\tau_{3} / m_{3} \geq 7.22 \times 10^{-13} \mathrm{~s} / \mathrm{eV}$ and $\tau_{3} / m_{3} \geq 1.41 \times 10^{-12} \mathrm{~s} / \mathrm{eV}$, respectively, while NOvA and T2K combined constrain $\tau_{3} / m_{3} \geq 1.50 \times 10^{-12} \mathrm{~s} / \mathrm{eV}$. We also show that in presence of decay the best-fit value in the $\sin ^{2} \theta_{23}$ vs. $\Delta m_{32}^{2}$ plane changes significantly and the allowed regions increase significantly towards higher $\sin ^{2} \theta_{23}$.

KeYwords: Neutrino Detectors and Telescopes (experiments), Oscillation

ARXIV EPRINT: 1805.01848 


\section{Contents}

1 Introduction 1

2 Invisible neutrino decay and simulation framework $\quad 4$

3 Results 5

4 Summary \& conclusion $\quad 10$

\section{Introduction}

The neutrino oscillation physics has entered its precision age. Among the six oscillation parameters associated with the standard neutrino oscillation physics, $\Delta m_{21}^{2}$ and $\theta_{12}$ have been measured precisely from the solar neutrino experiments [1] and KamLAND [2]. The atmospheric neutrino experiments [3] first showed evidence of $\nu_{\mu}-\nu_{\tau}$ flavor transformation and gave a measurement of $\left|\Delta m_{32}^{2}\right|$ and $\theta_{23}$. The mixing angle $\theta_{13}$ is also now precisely determined from reactor experiments DayaBay [4], Double CHOOZ [5] and RENO [6]. Currently the unknown quantities in the neutrino oscillation physics are the neutrino mass hierarchy/ordering i.e., whether the lightest neutrino state is $\nu_{1}$ or $\nu_{3}$, the precise measurement of $\theta_{23}$ and its octant and the CP-violating phase $\delta_{\mathrm{CP}}$. NOvA $[7,8]$ and T2K [9] are the presently running long baseline experiments. These are complimentary to the previous experiments and are expected to shed light on the unknown neutrino parameters.

The NOvA long-baseline experiment is in U.S.A. The $\nu_{\mu}$ flux is generated by the NuMI beam at Fermilab. The experiment employs two identical Totally Active Scintillator Detector (TASD) which are different only in terms of their mass. The Near detector (ND) is a $100 \mathrm{~m}$ deep 290 ton detector while the Far Detector (FD) is on the surface with mass 14 kton. The neutrino flux is measured first at the ND located at $1 \mathrm{~km}$ away from the target. The neutrino beam is next detected at the FD situated $810 \mathrm{~km}$ away near Ash River, Minnesota at an off-axis angle of $14.6 \mathrm{mrad}$. The $14.6 \mathrm{mrad}$ off-axis location of the FD gives a narrow energy spectrum at the FD with a peak near $2 \mathrm{GeV}$, which is tuned close to the first oscillation maximum at this baseline.

T2K (Tokai to Kamioka) is a long-baseline experiment in Japan. Here the $\nu_{\mu}$ beam is produced at the J-PARC accelerator complex in Japan by impinging a $30 \mathrm{GeV}$ proton beam onto a carbon target. This experiment also employs a two detector set-up that are off-axis compared to the beam direction. The neutrino produced from the beam are detected first at the ND, ND280, at $280 \mathrm{~m}$ from the target. The FD is the Super-Kamiokande detected with fiducial mass $22.5 \mathrm{kton}$ and situated $295 \mathrm{~km}$ away from the source at $2.5^{\circ}$ off-axis from the main beam axis, giving a narrow beam peaked at around $600 \mathrm{MeV}$, which for T2K's baseline corresponds to the first oscillation maximum. 
NOvA and T2K have both presented their initial results. The T2K experiment announced their first result with $1.43 \times 10^{20}$ protons on target (POT) on electron appearance in 2011 [10]. With six observed electron candidate events and 1.5 expected backgrounds, T2K gave the first direct evidence of non-zero $\theta_{13}$ at $2.5 \sigma$ C.L. The first results announcement on muon disappearance came a year later in 2012 [11] with the same POT of $1.43 \times 10^{20}$ and gave a best-fit of $\Delta m_{32}^{2}=2.65 \times 10^{-3} \mathrm{eV}^{2}$ and $\sin ^{2} 2 \theta_{23}=0.98$. The first anti-neutrino result from T2K was published in [12], where they used $\bar{\nu}_{\mu}$ beam with $4.01 \times 10^{20} \mathrm{POT}$ and obtained best fit of $\sin ^{2} \theta_{23}=0.45$ and $\Delta m_{32}^{2}=2.51 \times 10^{-3} \mathrm{eV}^{2}$, very consistent with the measurements obtained using the $\nu_{\mu}$ beam. The T2K collaboration has published their results periodically since the first announcements and their data-sets and best-fit oscillation parameters have remained consistent, with $\theta_{23}$ close to maximal. The latest result form T2K [13] used $7.482 \times 10^{20}$ POT for neutrino mode and $7.471 \times 10^{20}$ POT for antineutrino data. This currently gives the best fit $\Delta m_{32}^{2}=2.52 \pm 0.08(2.51 \pm 0.08) \times 10^{-3} \mathrm{eV}^{2}$ and $\sin ^{2} \theta_{23}=0.55_{-0.09}^{+0.05}\left(0.55_{0.08}^{+0.05}\right)$ for normal (inverted) ordering, using both electron appearance as well as muon disappearance data. The first result of muon-neutrino disappearance from NOvA came in 2016 [14], where they used $2.74 \times 10^{20}$ POT and got the best fit $\Delta m_{32}^{2}=\left(2.52_{-0.18}^{+0.20}\right) \times 10^{-3} \mathrm{eV}^{2}$ and $\sin ^{2} \theta_{23}=0.43$ and 0.60 . The was immediately followed with first result on electron appearance data [7] with the same exposure. Next disappearance data came in 2017 [15] which used $6.05 \times 10^{20}$ POT and gave $\Delta m_{32}^{2}=(2.67 \pm 0.11) \times 10^{-3} \mathrm{eV}^{2}$, while for $\sin ^{2} \theta_{23}$ they obtained two statistically degenerate values $0.404_{-0.022}^{+0.030}$ and $0.624_{-0.030}^{+0.022}$ and claimed that the NOvA data disfavours maximal mixing at $2.6 \sigma$. Results from the combined analysis of NOvA's appearance and disappearance data was presented in [8]. So while T2K prefers maximal mixing for $\theta_{23}$, the early analysis from the NOvA showed $2.6 \sigma$ preference for non-maximal mixing. This tension between the two experiments led several authors to propose new physics ideas to explain the tension between the two datasets. However, the NOvA collaboration has recently done an improved re-analysis of their disappearance dataset [16]. The newer analysis mainly addresses better the energy resolution of the hadron sample leading to an improved neutrino energy resolution. They have divided the muon events into four quantiles of different resolutions from $\sim 6 \%$ to $\sim 12 \%$, based on their hadronic energy fraction. This approach changed the measurement of $\theta_{23}$ at NOvA with maximal $\theta_{23}$ mixing being preferred by NOvA as well. Hence, the tension between NOvA and T2K has been resolved for now and the datasets seem to be consistent with standard three-generation flavor oscillations.

Although the data appears to be consistent with the standard expected three-generation paradigm, there can still be new physics effects present in these experiments. One such new physics scenario is neutrino decay. The active neutrino state could decay into another lighter active neutrino state and boson(s), or it could decay into a sterile fermion and boson(s). The former scenario is called visible neutrino decay [17-19] (since the final state fermion is active and hence "visible" to the detector) while the latter is known as invisible neutrino decay (since the final state fermion is sterile and hence "invisible" to the detector). The bosonic state(s) are assumed to be invisible in both class of models. In this paper we will consider neutrino decay into all invisible states only. There are two possibilities for such models: (i) The neutrino could decay into a Dirac fermion $[20,21]$ 
$\nu_{j} \rightarrow \bar{\nu}_{i R}+\chi$, where $\bar{\nu}_{i R}$ is a right-handed singlet and $\chi$ is an iso-singlet scalar carrying a lepton number. (ii) The neutrinos could decay into a Majorana fermion and a Majoron $\nu_{j} \rightarrow \nu_{s}+J[22,23]$. To evade the constraints of the $\mathrm{Z}$ decay to invisible particles from LEP data, the Majoron should be dominantly singlet [24]. First idea of decaying neutrinos was proposed very early in order to explain the solar neutrino problem [25]. Later neutrino oscillations along with decay solution to the solar neutrino problem was studied in [21, 2633]. These studies obtained bound on $\tau_{2}$ by considering $\nu_{2}$ to be unstable. The bound on $\tau_{2}$ from the solar data is $\tau_{2} / m_{2}>7.2 \times 10^{-4} \mathrm{~s} / \mathrm{eV}$ at $99 \%$ C.L. [32]. (See [31, 33] for other study.) The bound on $\nu_{e}$ lifetime from SN1987A are much more stringent [34]. Atmospheric and long-baseline (LBL) neutrinos give the bound on the $\nu_{3}$ lifetime. To solve the atmospheric neutrino problem a pure neutrino decay was proposed in [35], however this gave a very poor fit to the data. Authors of $[36,37]$ considered neutrino decay with mixing and claimed that it could somewhat reproduce the SK results, however, the zenith angle dependent SK data gave a poor fit [38]. In [36, 38] the $\Delta m^{2}$ dependent terms were averaged out. However, if the unstable neutrino state is allowed to decay into a sterile state with which it does not mix then the constraints on $\Delta m^{2}$ can be relaxed. Two scenarios have been studied in this context. In [39] $\Delta m^{2} \ll 10^{-4} \mathrm{eV}^{2}$ was considered and it was claimed to fit the data better, however the analysis by the SK collaboration showed that this gives poorer fit than the only oscillation case [40]. The second scenario was considered in [41], where $\Delta m^{2}$ was kept unconstrained. For SK data, it was shown that a small non-zero decay parameter and $\Delta m^{2} \sim 0.003 \mathrm{eV}^{2}$ gave a better fit to the data. The global analysis of atmospheric and MINOS data was performed in [42]. Although only oscillation gave the best-fit, but the decay plus oscillation scenario also gave a good fit. The bound obtained from the analysis is $\tau_{3} / m_{3} \geq 2.9 \times 10^{-10} \mathrm{~s} / \mathrm{eV}$ at the $90 \%$ C.L. Prospects of constraining the $\nu_{3}$ lifetime with atmospheric neutrino events at INO was studied in [43].

The case for visible neutrino decay in long-baseline experiments was considered for T2K and MINOS in [44] and DUNE in [45]. For T2K authors of [44] considered the charged current appearance and disappearance as well as neutral current data while for MINOS only the charged current data was taken. However, the visible neutrino decay scenario is very tightly constrained from data from other experiments. Therefore, we consider in this paper only the case for invisible neutrino decay. The analysis [46] considered MINOS and $\mathrm{T} 2 \mathrm{~K}$ data with two generation of neutrinos and gave the bound, $\tau_{3} / m_{3} \geq 2.8 \times 10^{-12} \mathrm{~s} / \mathrm{eV}$ at $90 \%$ C.L. The expected results at DUNE in the invisible neutrino decay scenario was worked out in [47].

In this paper we present the current constraints on $\tau_{3} / m_{3}$ from the recent data from NOvA and T2K, using the full three generation oscillation framework with matter effects. We also study the effect of decay on the measurement of $\theta_{23}$ and $\Delta m_{32}^{2}$. As was shown in $[46,47]$, there can be significant impact on the measured value of $\theta_{23}$ if decay is present. We will study how presence of decay changes the best-fit values of $\theta_{23}$ and $\Delta m_{32}^{2}$ as well as the C.L. contours allowed by the T2K and NOvA data.

The paper is organised as follows. In the next section we give the theoretical description of neutrino propagation when the $\nu_{3}$ state decays into a sterile state with which it does not mix. Also given in section 2 are the details of our simulation framework needed for the 
real data analysis of T2K and NOvA. We next give our result in section 3 and finally we conclude in section 4 .

\section{Invisible neutrino decay and simulation framework}

We assume that the $\nu_{3}$ state decays into a sterile neutrino and a singlet scalar $\left(\nu_{3} \rightarrow \overline{\nu_{4}}+J\right)$. We also assume that the neutrino mass eigenstate decays, therefore the mass matrix as well as the decay matrix can be simultaneously diagonalised. In this case the mixing between the flavor and the mass eigenstates can be written as,

$$
\left(\begin{array}{c}
\nu_{\alpha} \\
\nu_{s}
\end{array}\right)=\left(\begin{array}{ll}
U & 0 \\
0 & 1
\end{array}\right)\left(\begin{array}{l}
\nu_{i} \\
\nu_{4}
\end{array}\right)
$$

where the greek indices represent the standard flavor states i.e., $e, \mu, \tau$ and the latin indices represent the mass eigenstates. $U$ is the standard PMNS matrix. Here we assume NH i.e., $m_{3}>m_{2}>m_{1}$. In presence of decay the evolution equation in matter becomes:

$$
i \frac{d}{d x}\left(\begin{array}{c}
\nu_{e} \\
\nu_{\mu} \\
\nu_{\tau}
\end{array}\right)=\left[U\left[\frac{1}{2 E}\left(\begin{array}{ccc}
0 & 0 & 0 \\
0 & \Delta m_{21}^{2} & 0 \\
0 & 0 & \Delta m_{31}^{2}
\end{array}\right)-i \frac{\alpha_{3}}{2 E}\left(\begin{array}{lll}
0 & 0 & 0 \\
0 & 0 & 0 \\
0 & 0 & 1
\end{array}\right)\right] U^{\dagger}+\left(\begin{array}{ccc}
A & 0 & 0 \\
0 & 0 & 0 \\
0 & 0 & 0
\end{array}\right)\right]\left(\begin{array}{c}
\nu_{e} \\
\nu_{\mu} \\
\nu_{\tau}
\end{array}\right)
$$

where $A=2 \sqrt{2} G_{\mathrm{F}} n_{e} E$ represents the matter potential due to neutrino electron scattering in matter, $G_{\mathrm{F}}$ is the Fermi coupling, $E$ is the neutrino energy and $n_{e}$ is the electron density. We solve eq. (2.2) numerically using Runge-Kutta with constant matter density.

The simulation is done using a modified version of GLoBES, with modifications needed for real data analysis.

For the analysis of NOvA we have taken a $14 \mathrm{kt}$ detector at a baseline of $812 \mathrm{~km}$ with constant matter density. We have taken $8.5 \%$ energy resolution for electron events and $6 \%$ resolution for muon events. The signal efficiency is chosen to be $99 \%$ for electron events and $91 \%$ for muon events. We normalize the number of events as well as the shape, to match the best fit event spectra given in [8] for electrons $\left(6.04 \times 10^{20} \mathrm{POT}\right)$ and in [16] for muons $\left(8.85 \times 10^{20} \mathrm{POT}\right)$.

For the analysis of T2K, we have taken a $22.5 \mathrm{kt}$ detector at a baseline of $295 \mathrm{~km}$ with constant matter density. The energy resolution is taken $8.5 \%$. The signal efficiency is chosen to be $51.5 \%$ for electron events and $90 \%$ for muon events. We normalise the event spectra as well as shape, to match the event spectra given in [13] which corresponds to $7.482 \times 10^{20}$ POT in neutrino mode and $7.471 \times 10^{20}$ POT for anti-neutrino mode.

In all our analysis, we have assumed normal hierarchy. We have kept $\theta_{12}, \theta_{13}$ and $\Delta m_{21}^{2}$ fixed in the fit as these parameters are very precisely measured form the past experiments and we have marginalised over $\theta_{23}, \Delta m_{31}^{2}, \tau_{3} / m_{3}$ and $\delta_{\mathrm{CP}}$ as required. The ranges of the marginalisation are the following: for $\theta_{23}$ it is from $38^{\circ}$ to $53^{\circ}$, for $\Delta m_{31}^{2}$, it is from $2.3 \times 10^{-3} \mathrm{~s} / \mathrm{eV}^{2}$ to $2.6 \times 10^{-3} \mathrm{~s} / \mathrm{eV}^{2}$, for $\delta_{\mathrm{CP}}$, it is the full $360^{\circ}$ range and for $\tau_{3} / m_{3}$, the range is from $10^{-13} \mathrm{~s} / \mathrm{eV}$ to $10^{-9} \mathrm{~s} / \mathrm{eV}$. 


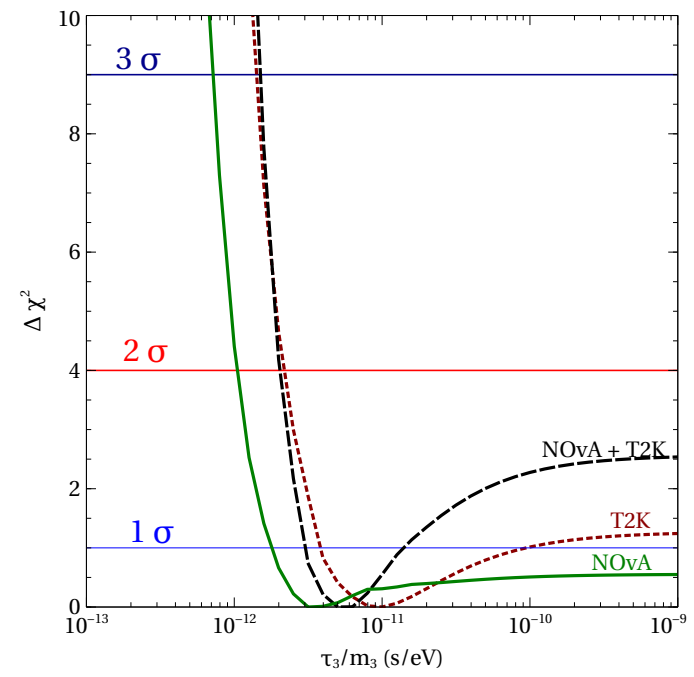

Figure 1. $\Delta \chi^{2}=\chi^{2}-\chi_{\min }^{2}$ vs. $\tau_{3} / m_{3}$ obtained from the analysis of T2K data (red dashed line), NOvA data (solid green line) and T2K+NOvA data (black long dashed line).

\section{Results}

In figure 1 we show the constraint on $\tau_{3} / m_{3}$ from the current data of NOvA and T2K, where the latest appearance as well as disappearance data sets of both experiments have been taken into consideration in the analysis. The green solid curve is obtained using NOvA data alone, the dark red dotted curve is obtained using T2K data alone, while the black dashed curve is obtained from a combined analysis of NOvA and T2K data. The parameters $\theta_{23}$ and $\Delta m_{32}^{2}$ are marginalised in the fit over their $3 \sigma$ allowed ranges and $\delta_{\mathrm{CP}}$ is marginalised over its full range. It can be seen from the figure 1 , that for both experiments the no decay scenario is slightly disfavoured and the best-fit value from the fit comes for non-zero decay. NOvA disfavors the no decay scenario at $0.7 \sigma$ and the best-fit value is $\tau_{3} / m_{3}=3.16 \times 10^{-12} \mathrm{~s} / \mathrm{eV}$. T2K disfavors the no decay case at slightly more than $1 \sigma$ and the best-fit value is $1 \times 10^{-11} \mathrm{~s} / \mathrm{eV}$. For the combined analysis case, the no decay case is disfavoured at $1.5 \sigma$ and the best-fit obtained from the combination of the two data is $\tau_{3} / m_{3}=5.01 \times 10^{-12} \mathrm{~s} / \mathrm{eV}$. The minimum $\chi^{2}\left(\chi_{\min }^{2}\right)$ for NOvA, T2K and the combined case are 10.38, 69.34 and 87.19, respectively, which are slightly less than the standard oscillation fit, for which the $\chi_{\min }^{2}$ are $10.93,70.39$ and 88.65 , respectively. Therefore, the invisible decay scenario we consider in this work, fits the data slightly better than the standard oscillation case. The data sets also set a lower bound on the lifetime. The $3 \sigma$ lower bound on $\tau_{3} / m_{3}$ from NOvA data is seen to be $\tau_{3} / m_{3} \geq 7.22 \times 10^{-13} \mathrm{~s} / \mathrm{eV}$, while from $\mathrm{T} 2 \mathrm{~K}$ it is $\tau_{3} / m_{3} \geq 1.41 \times 10^{-12} \mathrm{~s} / \mathrm{eV}$. The $3 \sigma$ combined constraint from both experiments taken together is $\tau_{3} / m_{3} \geq 1.50 \times 10^{-12} \mathrm{~s} / \mathrm{eV}$. It can be clearly seen from figure 1 , that the sum of the two $\Delta \chi^{2}$ is less than the $\Delta \chi^{2}$ for the combined analysis. This points at a synergy between the two experiments. This synergy results in an improved fit for the decay scenario compared to standard oscillation when we perform the combined analysis of the two experiments.

To understand why both the data prefer decay over standard oscillation, we give the muon event rates in figure 2. The left panel is for NOvA and the right panel is for T2K. 

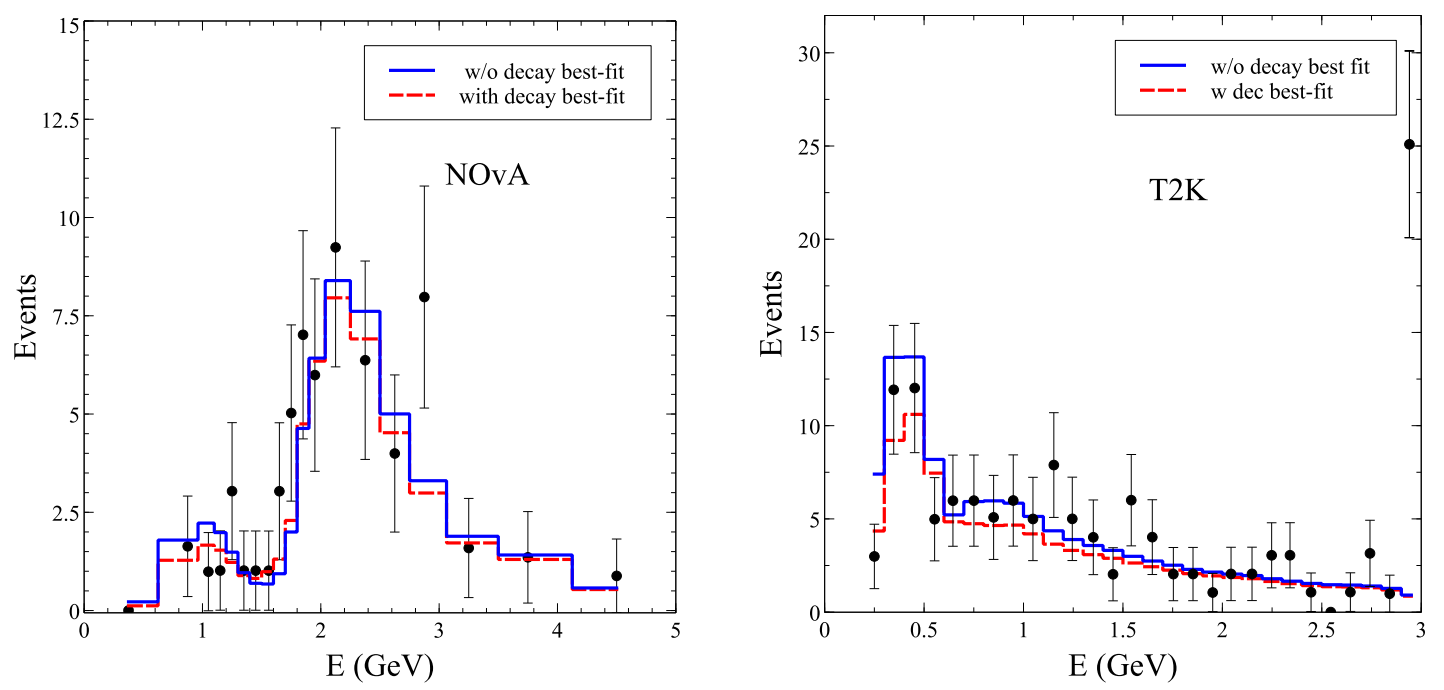

Figure 2. Muon event spectra for NOvA and T2K. The left panel is for NOvA while the right panel is for T2K. The black dots with the error-bar are actual data points. The error bars correspond to the $1 \sigma$ statistical error.

The blue solid lines show the event spectra for the standard best-bit, while the red dashed lines show the event spectra for decay best-fit. The black dots are data points with $1 \sigma$ statistical error bar. From the NOvA plot, it can be seen that the red dashed line fits the data better than the blue solid line. Between 1 and $2 \mathrm{GeV}$, where the oscillation maximum lies, the red dashed line almost exactly fits the data. For the T2K plot, again the red dashed line fits the data better than the blue solid line. Therefore, for both data sets, the decay with oscillation fits the data better than the only oscillation case.

Figure 3 shows the allowed region in the $\sin ^{2} \theta_{23}$ vs. $\Delta m_{32}^{2}$ plane at $95 \%$ C.L. The solid curves are for only oscillation case without decay whereas the dashed curves are for the case where $\nu_{3}$ are allowed to decay. The fit is marginalised over $\delta_{\mathrm{CP}}$ in both cases and over $\tau_{3} / m_{3}$ as well for the case of decay plus oscillation. The blue curves are for NOvA, the black curves are for T2K and the red curves are for the combined analysis. For the standard case the best-fit points $\left(\sin ^{2} \theta_{23}, \Delta m_{32}^{2}\right)$ are $\left(0.45,2.41 \times 10^{-3} \mathrm{eV}^{2}\right),\left(0.52,2.56 \times 10^{-3} \mathrm{eV}^{2}\right)$ and $\left(0.46,2.51 \times 10^{-3} \mathrm{eV}^{2}\right)$ for NOvA, T2K and the combined cases, respectively. On the other hand, for the case with decay and oscillation the corresponding best-fit points are: $\left(0.48,2.39 \times 10^{-3} \mathrm{eV}^{2}\right),\left(0.62,2.62 \times 10^{-3} \mathrm{eV}^{2}\right)$ and $\left(0.48,2.52 \times 10^{-3} \mathrm{eV}^{2}\right)$ for NOvA, T2K and the combined cases, respectively. The interesting point to notice here is that for all cases, the allowed region of the parameter space increases significantly when decay is considered along with oscillation. Also note that with inclusion of decay, the best-fit shifts towards higher values of $\sin ^{2} \theta_{23}$. This behaviour is very similar to what was seen in [46] in the context of MINOS and T2K and in [47] in the context of DUNE. The shift of $\theta_{23}$ to higher values in presence of decay can be understood in terms of the survival probability given in [46] in the two-generation approximation neglecting matter effect,

$P_{\mu \mu}^{2 G}=\left[\cos ^{2} \theta_{23}+\sin ^{2} \theta_{23} \exp \left(-m_{3} L / \tau_{3} E\right)\right]^{2}-\sin ^{2} 2 \theta_{23} \exp \left(-m_{3} L / \tau_{3} E\right) \sin ^{2}\left(\frac{\Delta m_{31}^{2} L}{4 E}\right)$. 


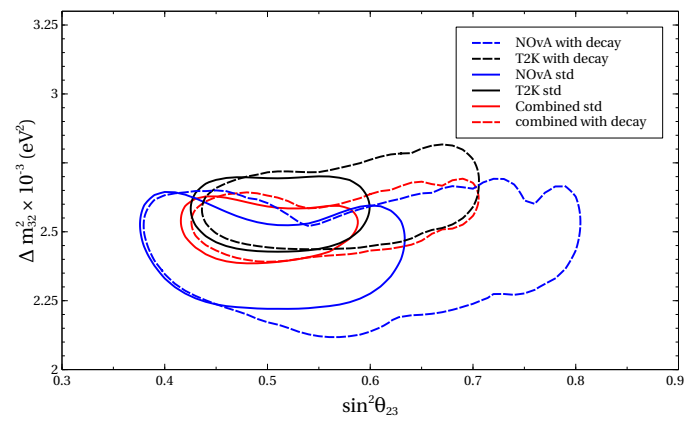

Figure 3. The $95 \%$ C.L. allowed areas obtained in the $\sin ^{2} \theta_{23}-\Delta m_{32}^{2}$ plane, from analysis of T2K data (red lines), NOvA data (green lines) and T2K+NOvA data (black lines). The solid lines are for standard three-generation oscillations while the dashed lines are for oscillation with decay.

In eq. (3.1), there is an exponential suppression due to neutrino decay in both the oscillatory as well as the non-oscillatory term. Therefore for a given $\theta_{23}$, the survival probability for the decay case will be less than the standard oscillation case. Hence, when decay is considered in the fit, the value of $\sin ^{2} \theta_{23}$ increases in order to reproduce the same probability obtained for the standard case.

From figure 4, one can understand why more region of higher octant is allowed than the lower octant for the case with decay. The left(right) panel of figure 4 show the muon events for $\operatorname{NOvA}(\mathrm{T} 2 \mathrm{~K})$. In each of the plots, the blue solid line is for $\sin ^{2} \theta_{23}$ in higher octant and the red dashed line is for $\sin ^{2} \theta_{23}$ in the lower octant. In each case decay is present. The black dots are the data points with $1 \sigma$ statistical error bar. In both of the plots, it can be seen that the blue solid lines fit the data points reasonably whereas the red dashed lines do not fit the data at all. This shows why more region of the higher octant is allowed for the decay case than the lower octant. This is consistent with eq. (3.1) where we can see that for a given $\sin ^{2} 2 \theta_{23}$, the survival probability is significantly larger for the lower octant compared to the higher octant. Since both NOvA and T2K disappearance data are consistent with a suppressed muon neutrino survival probability, presence of decay allows larger allowed areas in the higher octant of $\theta_{23}$ compared to the standard oscillation case, while in the lower octant the contours shift marginally to the left.

Figure 5 gives the allowed region in the $\sin ^{2} \theta_{23}$ vs. $\tau_{3} / m_{3}$ plane at $95 \%$ C.L. with $\Delta m_{32}^{2}$ marginalised over its current $3 \sigma$ range and $\delta_{\mathrm{CP}}$ marginalised over full range. The blue solid, black dashed and red dashed-dotted curves are for NOvA, T2K and the combined analysis, respectively. The blue cross gives the best-fit for NOvA $\left(0.47,3.16 \times 10^{-12} \mathrm{~s} / \mathrm{eV}\right)$, the black plus gives the best-fit for T2K $\left(0.61,5.011 \times 10^{-12} \mathrm{~s} / \mathrm{eV}\right)$ and the red star gives the best-fit for the combined case $\left(0.49,5.011 \times 10^{-12} \mathrm{~s} / \mathrm{eV}\right)$. Again as in figure 3, the best-fit is seen to be for finite $\tau_{3} / m_{3}$.

Figure 6 gives the $\Delta \chi^{2}$ vs. $\sin ^{2} \theta_{23}$ for the standard case and the decay plus oscillation case. The fit is marginalised over $\Delta m_{32}^{2}$ and $\delta_{\mathrm{CP}}$ for the standard case and over $\Delta m_{32}^{2}$, $\tau_{3} / m_{3}$ and $\delta_{\mathrm{CP}}$ for the decay plus oscillation case. The left panel is for NOvA, the middle panel is for $\mathrm{T} 2 \mathrm{~K}$ and the right panel is for the combined analysis. In all the panels the blue solid curves are for the standard oscillation case while the red dashed curves represent 

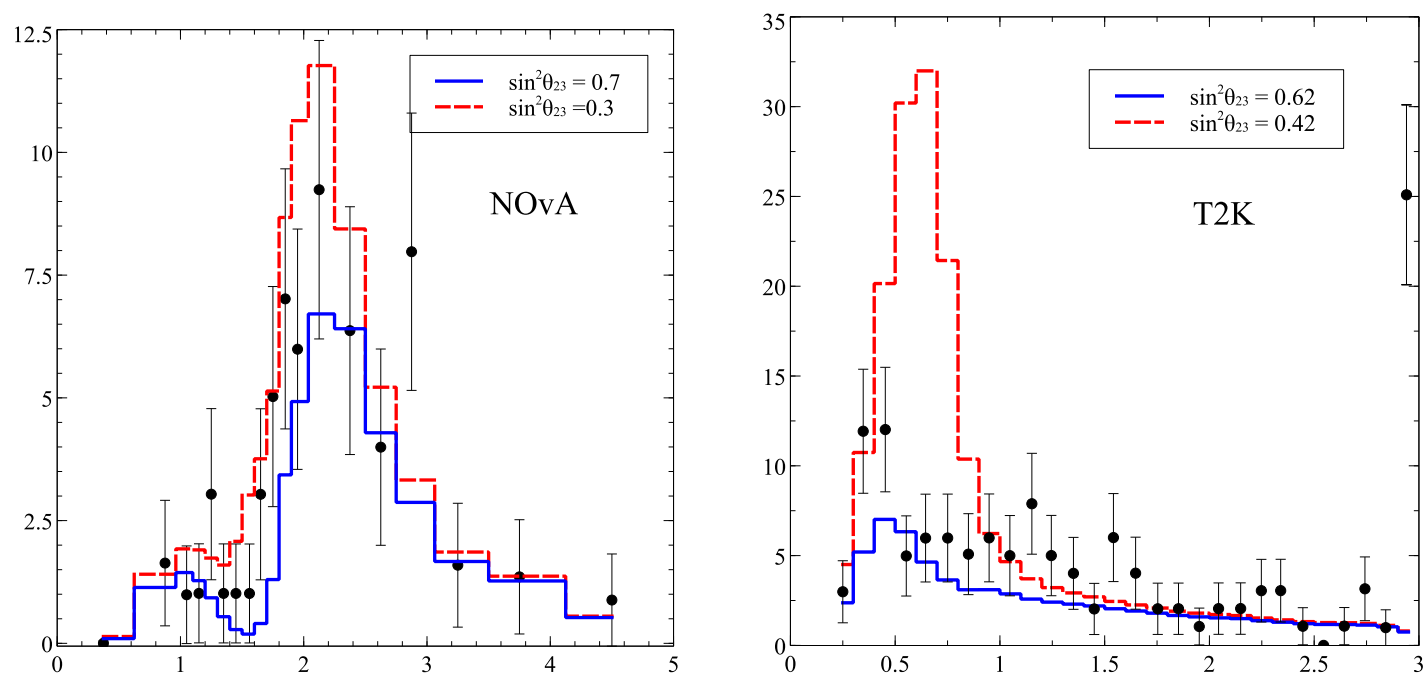

Figure 4. Muon event spectra for NOvA and T2K. The left panel is for NOvA while the right panel is for T2K. The black dots with the error-bar are actual data points. The error bars correspond to the $1 \sigma$ statistical error.

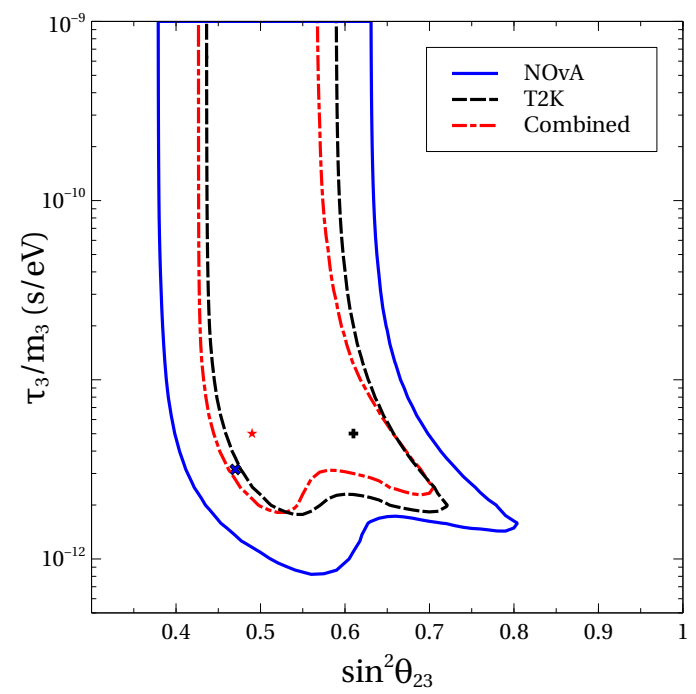

Figure 5. The 95\% C.L. allowed areas obtained in the $\tau_{3} / m_{3}-\sin ^{2} \theta_{23}$ plane, from analysis of T2K data (black dashed line), NOvA data (blue solid line) and T2K+NOvA data (red dasheddotted line).

the decay with oscillation case. For T2K our standard oscillation best-fit $\sin ^{2} \theta_{23}=0.52$ matches very well with the best-fit obtained by the T2K collaboration [13]. For NOvA on the other hand, our best-fit for standard oscillation comes at $\sin ^{2} \theta_{23}=0.45$ while the NOvA collaboration gets their best-fit at $\sin ^{2} \theta_{23}=0.558_{-0.033}^{+0.041}$ in the higher octant. The reason for this mild mis-match could be because our experimental simulation is based on GLoBES which cannot include all systematics in a rigorous manner. However, the figure shows that the $\chi^{2}$ differences between the minima of the two octant is less than 1 , and hence the best-fit obtained by us for the higher octant is not so different from the correct value obtained from the collaboration. 

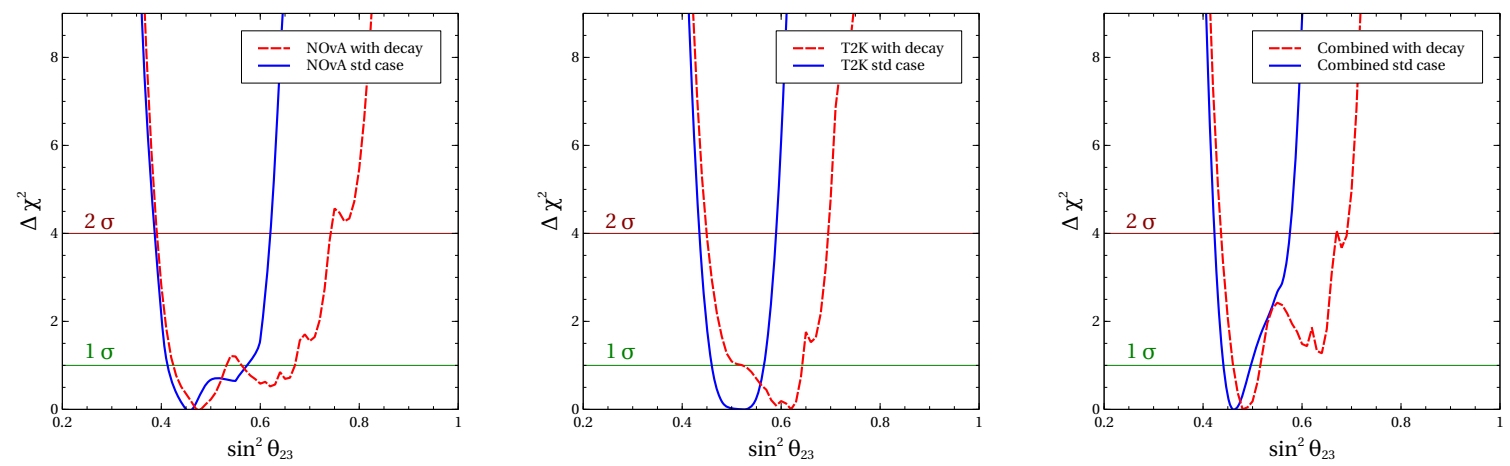

Figure 6. $\Delta \chi^{2}$ vs. $\sin ^{2} \theta_{23}$, where the $\chi^{2}$ is marginalised over all other parameters.

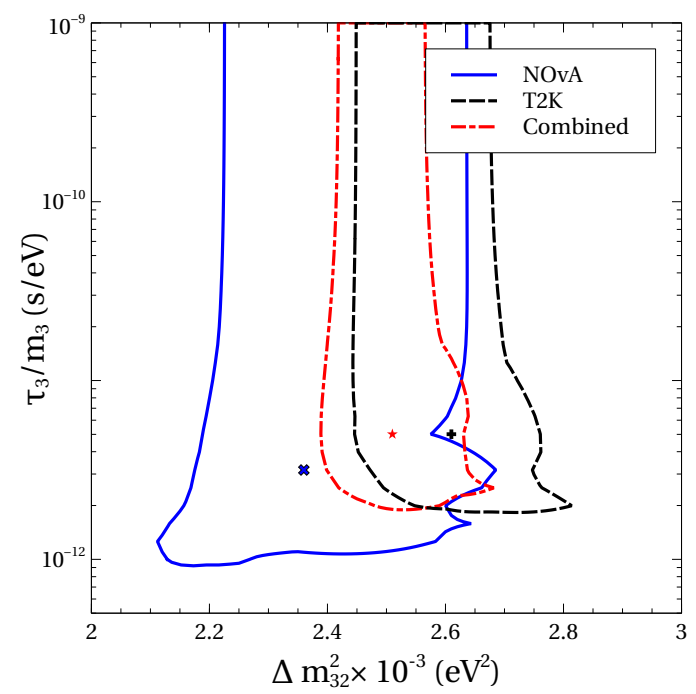

Figure 7. The $95 \%$ C.L. allowed areas obtained in the $\tau_{3} / m_{3}-\Delta m_{32}^{2}$ plane, from analysis of T2K data (black dashed line), NOvA data (blue solid line) and T2K+NOvA data (red dasheddotted line).

Figure 7 gives the allowed region in the $\Delta m_{32}^{2}$ vs. $\tau_{3} / m_{3}$ plane at $95 \%$ C.L. with $\theta_{23}$ marginalised over current $3 \sigma$ allowed region and $\delta_{\mathrm{CP}}$ marginalised over full range. The blue curve is for NOvA, the black is for T2K and the red is for the combined case. The blue cross gives the best-fit for NOvA $\left(2.36 \times 10^{-3}, 3.16 \times 10^{-12}\right)$, the black plus for the T2K $\left(2.61 \times 10^{-3}, 5.011 \times 10^{-12}\right)$ and the red star is for the combined best-fit $\left(2.51 \times 10^{-3}\right.$, $\left.5.011 \times 10^{-12}\right)$. The above best-fit values are in units of $\left(\mathrm{eV}^{2}, \mathrm{~s} / \mathrm{eV}\right)$.

Figure 8 gives the $\Delta \chi^{2}$ vs. $\Delta m_{32}^{2}$ with $\theta_{23}$ and $\delta_{\mathrm{CP}}$ marginalised for the standard and $\theta_{23}, \delta_{\mathrm{CP}}$ and $\tau_{3} / m_{3}$ marginalised for the case of decay plus oscillation. Just as in figure 6 , the left panel is for NOvA, the middle is for $\mathrm{T} 2 \mathrm{~K}$ and the right panel is for the combined case. The blue solid curves are for the standard case whereas the red dashed curves are for the decay plus oscillation case. It can be seen from the figure that for NOvA the allowed range of $\Delta m_{32}^{2}$ increases on both sides while for T2K the best-fit $\Delta m_{32}^{2}$ shifts towards higher values and as a result the allowed range shifts towards the right.

To check the compatibility of the two data sets, we have calculated the parameter goodness of fit [48] for $\theta_{23}$ and $\Delta m_{32}^{2}$, with both hypotheses. For $\theta_{23}$, the parameter 

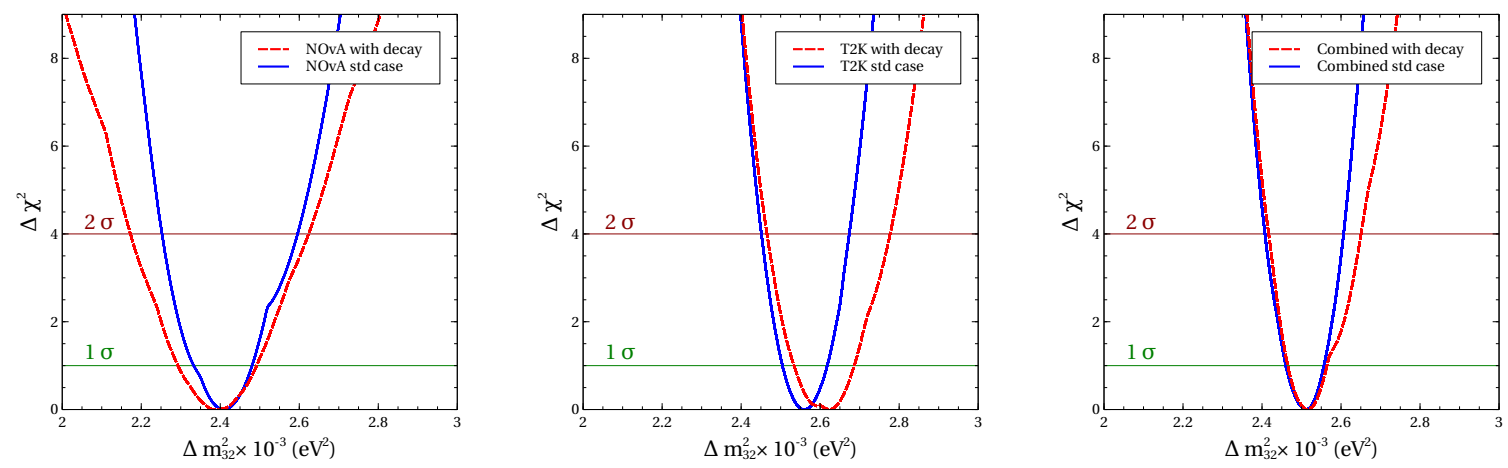

Figure 8. $\Delta \chi^{2}$ vs. $\Delta m_{32}^{2}$, where the $\chi^{2}$ is marginalised over all other parameters.

goodness of fit is $90 \%$ for standard oscillations and $97 \%$ for the decay case. For the measurement of $\Delta m_{32}^{2}$, the parameter goodness of fit is $43 \%$ for the standard case and $57 \%$ for the decay case. For $\sin ^{2} \theta_{23}$, the $\bar{\chi}_{\text {min }}^{2}$ is 0.56 for standard oscillation and 0.52 for the case of decay, where $\bar{\chi}^{2}$ is the sum of the $\chi^{2}$ of the two experiments as defined in [48]. The standard case has 3 free parameters $\left(\theta_{23}, \delta m_{32}^{2}\right.$ and $\left.\delta_{\mathrm{CP}}\right)$ whereas the decay case has $4\left(\theta_{23}, \delta m_{32}^{2}, \delta_{\mathrm{CP}}\right.$ and $\left.\tau_{3} / m_{3}\right)$. As the decay case has $\bar{\chi}_{\text {min }}^{2}$ less than the standard case as well as it has more free parameters, the parameter goodness of fit improves for the decay for $\sin ^{2} \theta_{23}$. For $\Delta m_{32}^{2}$, the $\bar{\chi}_{\text {min }}^{2}$ is 2.73 for the case of standard oscillations and 2.90 for the case of decay. Here, although the $\bar{\chi}_{\min }^{2}$ is more for the decay case but since decay case has more free parameters than the standard oscillation case, the parameter goodness of fit improves for the decay case.

\section{Summary \& conclusion}

We analysed the recent NOvA and T2K data for invisible decay of the neutrino. We considered a framework where the $\nu_{3}$ is unstable and it decays into some lighter sterile state with which the active neutrinos do not mix. We used modified GLoBES for simulating the $\mathrm{T} 2 \mathrm{~K}$ and NOvA experiments taking into account the experimental exposure, systematic uncertainties and resolution functions for the current data sets. We considered the latest disappearance and appearance data given by the $\mathrm{T} 2 \mathrm{~K}$ collaboration which corresponds to $7.482 \times 10^{20}$ POT for neutrino and $7.471 \times 10^{20}$ POT for anti-neutrinos [13]. For NOvA we consider the disappearance data and simulation framework announced by the collaboration in [16] and appearance data presented in [8]. To obtained the oscillation probabilities we considered the full three generation framework with matter effects and decay and solved the evolution equation for the neutrinos numerically. We performed a two-pronged analysis. On one hand we looked at how compatible the current T2K and NOvA data are with neutrino decay. On the other hand we checked how the best-fit values and allowed ranges on $\Delta m_{32}^{2}$ and $\sin ^{2} \theta_{23}$ change when decay is included in the fit.

We found that both $\mathrm{T} 2 \mathrm{~K}$ and NOvA data give better fit when neutrino decay in included. The best-fit lifetime corresponding to the T2K data is $\tau_{3} / m_{3}=1.0 \times 10^{-11} \mathrm{~s} / \mathrm{eV}$. This can be compared with the best-fit lifetime of $\tau_{3} / m_{3}=1.6 \times 10^{-12} \mathrm{~s} / \mathrm{eV}$ obtained in [46] 
using the older T2K data [49] which corresponded to $6.57 \times 10^{20}$ POT for the neutrino mode and did not have the anti-neutrino data. The main difference between the analysis performed in [46] and this work is the use of the anti-neutrino data, more exposure in the neutrino data and the inclusion of the electron appearance data. Our best-fit can also be compared to the best-fit lifetime of $\tau_{3} / m_{3}=1.2 \times 10^{-12} \mathrm{~s} / \mathrm{eV}$ obtained in [46] from the combined fit of $\mathrm{T} 2 \mathrm{~K}$ and MINOS data. The best-fit $\nu_{3}$ lifetime from NOvA data corresponds to $\tau_{3} / m_{3}=3.16 \times 10^{-12} \mathrm{~s} / \mathrm{eV}$, which is about an order of magnitude smaller than the T2K best-fit. The combined fit of T2K and NOvA returns a best-fit $\tau_{3} / m_{3}=5.01 \times 10^{-12} \mathrm{~s} / \mathrm{eV}$. The datasets also put a lower bound on the $\nu_{3}$ lifetime. The $3 \sigma$ bound put by T2K, NOvA and T2K+NOvA are $\tau_{3} / m_{3} \geq 1.41 \times 10^{-12} \mathrm{~s} / \mathrm{eV}, \tau_{3} / m_{3} \geq$ $7.22 \times 10^{-13} \mathrm{~s} / \mathrm{eV}$ and $\tau_{3} / m_{3} \geq 1.50 \times 10^{-12} \mathrm{~s} / \mathrm{eV}$, respectively.

We also studied the effect of decay on the measurement of the standard parameters $\theta_{23}$ and $\Delta m_{32}^{2}$. We found that if we include decay in our fit, the best-fit values for $\sin ^{2} \theta_{23}$ and $\Delta m_{32}^{2}$ change significantly. The best-fit $\left(\sin ^{2} \theta_{23}, \Delta m_{32}^{2}\right)$ obtained for the standard oscillation case from analysis of NOvA, T2K and both experiments combined are $(0.45$, $\left.2.41 \times 10^{-3} \mathrm{eV}^{2}\right),\left(0.52,2.56 \times 10^{-3} \mathrm{eV}^{2}\right)$ and $\left(0.46,2.51 \times 10^{-3} \mathrm{eV}^{2}\right)$ respectively. On including decay in the fit, the corresponding best-fit points become $\left(0.48,2.39 \times 10^{-3} \mathrm{eV}^{2}\right)$ for NOvA, $\left(0.62,2.62 \times 10^{-3} \mathrm{eV}^{2}\right)$ for T2K and $\left(0.48,2.52 \times 10^{-3} \mathrm{eV}^{2}\right)$ for the NOvA and T2K combined. The best-fit $\sin ^{2} \theta_{23}$ is seen to be shifting to higher values. We also give the $95 \%$ C.L. contours in the two-parameter space and the $\Delta \chi^{2}$ vs. $\sin ^{2} \theta_{23}$ and $\Delta \chi^{2}$ vs. $\Delta m_{32}^{2}$ plots from which 1,2 and $3 \sigma$ ranges of these parameters can be read for both hypothesis, with and without decay. Decay is seen to shift the allowed range of $\sin ^{2} \theta_{23}$ significantly to higher values, thereby extending the allowed ranges in the higher octant. The reason for this behavior was discussed. The allowed range of $\Delta m_{32}^{2}$ is also seen to change with inclusion of delay, albeit very mildly.

In conclusion, both $\mathrm{T} 2 \mathrm{~K}$ and NOvA, and in particular NOvA, seem to favor neutrino decay. Even though this conclusion is not statistically significant yet, it will be interesting to see the results from the forthcoming next-generation long baseline experiments like DUNE and T2HK. Invisible neutrino decay also results in shifting $\theta_{23}$ to higher values and this would be again an interesting phenomenon to study at the next-generation experiments.

\section{Acknowledgments}

We acknowledge the HRI cluster computing facility (http://www.hri.res.in/cluster/). This project has received funding from the European Union's Horizon 2020 research and innovation programme InvisiblesPlus RISE under the Marie Sklodowska-Curie grant agreement No. 690575. This project has received funding from the European Union's Horizon 2020 research and innovation programme Elusives ITN under the Marie Sklodowska- Curie grant agreement No. 674896 .

Open Access. This article is distributed under the terms of the Creative Commons Attribution License (CC-BY 4.0), which permits any use, distribution and reproduction in any medium, provided the original author(s) and source are credited. 


\section{References}

[1] SNO collaboration, Q.R. Ahmad et al., Direct evidence for neutrino flavor transformation from neutral current interactions in the Sudbury Neutrino Observatory, Phys. Rev. Lett. 89 (2002) 011301 [nucl-ex/0204008] [INSPIRE].

[2] KamLAND collaboration, A. Gando et al., Constraints on $\theta_{13}$ from A Three-Flavor Oscillation Analysis of Reactor Antineutrinos at KamLAND, Phys. Rev. D 83 (2011) 052002 [arXiv:1009.4771] [INSPIRE].

[3] Super-Kamiokande collaboration, Y. Fukuda et al., Evidence for oscillation of atmospheric neutrinos, Phys. Rev. Lett. 81 (1998) 1562 [hep-ex/9807003] [INSPIRE].

[4] DAYA BAy collaboration, F.P. An et al., Observation of electron-antineutrino disappearance at Daya Bay, Phys. Rev. Lett. 108 (2012) 171803 [arXiv:1203.1669] [INSPIRE].

[5] Double CHOOZ collaboration, Y. Abe et al., Indication of Reactor $\bar{\nu}_{e}$ Disappearance in the Double CHOOZ Experiment, Phys. Rev. Lett. 108 (2012) 131801 [arXiv:1112.6353] [INSPIRE].

[6] RENO collaboration, J.K. Ahn et al., Observation of Reactor Electron Antineutrino Disappearance in the RENO Experiment, Phys. Rev. Lett. 108 (2012) 191802 [arXiv: 1204.0626] [INSPIRE].

[7] NOvA collaboration, P. Adamson et al., First measurement of electron neutrino appearance in NOvA, Phys. Rev. Lett. 116 (2016) 151806 [arXiv:1601.05022] [INSPIRE].

[8] NOvA collaboration, P. Adamson et al., Constraints on Oscillation Parameters from $\nu_{e}$ Appearance and $\nu_{\mu}$ Disappearance in NOvA, Phys. Rev. Lett. 118 (2017) 231801 [arXiv:1703.03328] [INSPIRE].

[9] T2K collaboration, K. Abe et al., Combined Analysis of Neutrino and Antineutrino Oscillations at T2K, Phys. Rev. Lett. 118 (2017) 151801 [arXiv:1701.00432] [InSPIRE].

[10] T2K collaboration, K. Abe et al., Indication of Electron Neutrino Appearance from an Accelerator-produced Off-axis Muon Neutrino Beam, Phys. Rev. Lett. 107 (2011) 041801 [arXiv:1106.2822] [INSPIRE].

[11] T2K collaboration, K. Abe et al., First Muon-Neutrino Disappearance Study with an Off-Axis Beam, Phys. Rev. D 85 (2012) 031103 [arXiv:1201.1386] [InSPIRE].

[12] T2K collaboration, K. Abe et al., Measurement of Muon Antineutrino Oscillations with an Accelerator-Produced Off-Axis Beam, Phys. Rev. Lett. 116 (2016) 181801 [arXiv: 1512.02495] [INSPIRE].

[13] T2K collaboration, K. Abe et al., Measurement of neutrino and antineutrino oscillations by the T2K experiment including a new additional sample of $\nu_{e}$ interactions at the far detector, Phys. Rev. D 96 (2017) 092006 [arXiv:1707.01048] [INSPIRE].

[14] NOvA collaboration, P. Adamson et al., First measurement of muon-neutrino disappearance in NOvA, Phys. Rev. D 93 (2016) 051104 [arXiv: 1601.05037] [InSPIRE].

[15] NOvA collaboration, P. Adamson et al., Measurement of the neutrino mixing angle $\theta_{23}$ in NOvA, Phys. Rev. Lett. 118 (2017) 151802 [arXiv: 1701.05891] [INSPIRE].

[16] A. Radovic, Latest Oscillation results from NOvA, talk given at Fermilab, Batavia, IL, U.S.A., 12 Januray 2018. 
[17] C.W. Kim and W.P. Lam, Some remarks on neutrino decay via a Nambu-Goldstone boson, Mod. Phys. Lett. A 5 (1990) 297 [inSPIRE].

[18] A. Acker, A. Joshipura and S. Pakvasa, A Neutrino decay model, solar anti-neutrinos and atmospheric neutrinos, Phys. Lett. B 285 (1992) 371 [inSPIRE].

[19] M. Lindner, T. Ohlsson and W. Winter, A Combined treatment of neutrino decay and neutrino oscillations, Nucl. Phys. B 607 (2001) 326 [hep-ph/0103170] [InSPIRE].

[20] A. Acker, S. Pakvasa and J.T. Pantaleone, Decaying Dirac neutrinos, Phys. Rev. D 45 (1992) 1 [INSPIRE].

[21] A. Acker and S. Pakvasa, Solar neutrino decay, Phys. Lett. B 320 (1994) 320 [hep-ph/9310207] [INSPIRE].

[22] G.B. Gelmini and M. Roncadelli, Left-Handed Neutrino Mass Scale and Spontaneously Broken Lepton Number, Phys. Lett. B 99 (1981) 411 [INSPIRE].

[23] Y. Chikashige, R.N. Mohapatra and R.D. Peccei, Are There Real Goldstone Bosons Associated with Broken Lepton Number?, Phys. Lett. B 98 (1981) 265 [INSPIRE].

[24] S. Pakvasa, Do neutrinos decay?, AIP Conf. Proc. 542 (2000) 99 [hep-ph/0004077] [INSPIRE].

[25] J.N. Bahcall, N. Cabibbo and A. Yahil, Are neutrinos stable particles?, Phys. Rev. Lett. 28 (1972) 316 [INSPIRE].

[26] Z.G. Berezhiani, G. Fiorentini, M. Moretti and A. Rossi, Fast neutrino decay and solar neutrino detectors, Z. Phys. C 54 (1992) 581 [InSPIRE].

[27] Z.G. Berezhiani, M. Moretti and A. Rossi, Matter induced neutrino decay and solar anti-neutrinos, Z. Phys. C 58 (1993) 423 [INSPIRE].

[28] S. Choubey, S. Goswami and D. Majumdar, Status of the neutrino decay solution to the solar neutrino problem, Phys. Lett. B 484 (2000) 73 [hep-ph/0004193] [INSPIRE].

[29] A. Bandyopadhyay, S. Choubey and S. Goswami, MSW mediated neutrino decay and the solar neutrino problem, Phys. Rev. D 63 (2001) 113019 [hep-ph/0101273] [INSPIRE].

[30] A.S. Joshipura, E. Masso and S. Mohanty, Constraints on decay plus oscillation solutions of the solar neutrino problem, Phys. Rev. D 66 (2002) 113008 [hep-ph/0203181] [INSPIRE].

[31] A. Bandyopadhyay, S. Choubey and S. Goswami, Neutrino decay confronts the SNO data, Phys. Lett. B 555 (2003) 33 [hep-ph/0204173] [INSPIRE].

[32] R. Picoreti, M.M. Guzzo, P.C. de Holanda and O.L.G. Peres, Neutrino Decay and Solar Neutrino Seasonal Effect, Phys. Lett. B 761 (2016) 70 [arXiv:1506.08158] [INSPIRE].

[33] J.M. Berryman, A. de Gouvêa and D. Hernandez, Solar Neutrinos and the Decaying Neutrino Hypothesis, Phys. Rev. D 92 (2015) 073003 [arXiv:1411.0308] [InSPIRE].

[34] J.A. Frieman, H.E. Haber and K. Freese, Neutrino mixing, decays and supernova 1987A, Phys. Lett. B 200 (1988) 115 [inSPIRE].

[35] J.M. LoSecco, What the atmospheric neutrino anomaly is not, hep-ph/9809499 [INSPIRE].

[36] V.D. Barger, J.G. Learned, S. Pakvasa and T.J. Weiler, Neutrino decay as an explanation of atmospheric neutrino observations, Phys. Rev. Lett. 82 (1999) 2640 [astro-ph/9810121] [INSPIRE]. 
[37] P. Lipari and M. Lusignoli, On exotic solutions of the atmospheric neutrino problem, Phys. Rev. D 60 (1999) 013003 [hep-ph/9901350] [INSPIRE].

[38] G.L. Fogli, E. Lisi, A. Marrone and G. Scioscia, Super-Kamiokande data and atmospheric neutrino decay, Phys. Rev. D 59 (1999) 117303 [hep-ph/9902267] [INSPIRE].

[39] V.D. Barger, J.G. Learned, P. Lipari, M. Lusignoli, S. Pakvasa and T.J. Weiler, Neutrino decay and atmospheric neutrinos, Phys. Lett. B 462 (1999) 109 [hep-ph/9907421] [INSPIRE].

[40] Super-Kamiokande collaboration, Y. Ashie et al., Evidence for an oscillatory signature in atmospheric neutrino oscillation, Phys. Rev. Lett. 93 (2004) 101801 [hep-ex/0404034] [INSPIRE].

[41] S. Choubey and S. Goswami, Is neutrino decay really ruled out as a solution to the atmospheric neutrino problem from Super-Kamiokande data?, Astropart. Phys. 14 (2000) 67 [hep-ph/9904257] [INSPIRE].

[42] M.C. Gonzalez-Garcia and M. Maltoni, Status of Oscillation plus Decay of Atmospheric and Long-Baseline Neutrinos, Phys. Lett. B 663 (2008) 405 [arXiv:0802.3699] [INSPIRE].

[43] S. Choubey, S. Goswami, C. Gupta, S.M. Lakshmi and T. Thakore, Sensitivity to neutrino decay with atmospheric neutrinos at the INO-ICAL detector, Phys. Rev. D 97 (2018) 033005 [arXiv: 1709.10376] [INSPIRE].

[44] A.M. Gago, R.A. Gomes, A.L.G. Gomes, J. Jones-Perez and O.L.G. Peres, Visible neutrino decay in the light of appearance and disappearance long baseline experiments, JHEP 11 (2017) 022 [arXiv: 1705.03074] [INSPIRE].

[45] P. Coloma and O.L.G. Peres, Visible neutrino decay at DUNE, arXiv:1705.03599 [INSPIRE].

[46] R.A. Gomes, A.L.G. Gomes and O.L.G. Peres, Constraints on neutrino decay lifetime using long-baseline charged and neutral current data, Phys. Lett. B 740 (2015) 345 [arXiv: 1407.5640] [INSPIRE].

[47] S. Choubey, S. Goswami and D. Pramanik, A study of invisible neutrino decay at DUNE and its effects on $\theta_{23}$ measurement, JHEP 02 (2018) 055 [arXiv:1705.05820] [INSPIRE].

[48] M. Maltoni and T. Schwetz, Testing the statistical compatibility of independent data sets, Phys. Rev. D 68 (2003) 033020 [hep-ph/0304176] [INSPIRE].

[49] T2K collaboration, K. Abe et al., Precise Measurement of the Neutrino Mixing Parameter $\theta_{23}$ from Muon Neutrino Disappearance in an Off-Axis Beam, Phys. Rev. Lett. 112 (2014) 181801 [arXiv: 1403.1532] [INSPIRE]. 